\title{
Mean-Square Performance Analysis of the Family of Selective Partial Update NLMS and Affine Projection Adaptive Filter Algorithms in Nonstationary Environment
}

\author{
Mohammad Shams Esfand Abadi and Fatemeh Moradiani \\ Faculty of Electrical and Computer Engineering, Shahid Rajaee Teacher Training University, P.O. Box 16785-163, Tehran, Iran \\ Correspondence should be addressed to Mohammad Shams Esfand Abadi, mshams@srttu.edu
}

Received 30 June 2010; Revised 29 August 2010; Accepted 11 October 2010

Academic Editor: Antonio Napolitano

Copyright (c) 2011 M. Shams Esfand Abadi and F. Moradiani. This is an open access article distributed under the Creative Commons Attribution License, which permits unrestricted use, distribution, and reproduction in any medium, provided the original work is properly cited.

\begin{abstract}
We present the general framework for mean-square performance analysis of the selective partial update affine projection algorithm (SPU-APA) and the family of SPU normalized least mean-squares (SPU-NLMS) adaptive filter algorithms in nonstationary environment. Based on this the tracking performance of Max-NLMS, N-Max NLMS and the various types of SPU-NLMS and SPU-APA can be analyzed in a unified way. The analysis is based on energy conservation arguments and does not need to assume a Gaussian or white distribution for the regressors. We demonstrate through simulations that the derived expressions are useful in predicting the performances of this family of adaptive filters in nonstationary environment.
\end{abstract}

\section{Introduction}

Mean-square performance analysis of adaptive filtering algorithms in nonstationary environments has been, and still is, an area of active research [1-3]. When the input signal properties vary with time, the adaptive filters are able to track these variations. The aim of tracking performance analysis is to characterize this tracking ability in nonstationary environments. In this area, many contributions focus on a particular algorithm, making more or less restrictive assumptions on the input signal. For example, in $[4,5]$, the transient performance of the LMS was presented in the nonstationary environments. The former uses a randomwalk model for the variations in the optimal weight vector, while the latter assumes deterministic variations in the optimal weight vector. The steady-state performance of this algorithm in the nonstationary environment for the white input is presented in [6]. The tracking performance analysis of the signed regressor LMS algorithm can be found in [7-9]. Also, the steady-state and tracking analysis of this algorithm without the explicit use of the independence assumptions are presented in [10].
Obviously, a more general analysis encompassing as many different algorithms as possible as special cases, while at the same time making as few restrictive assumptions as possible, is highly desirable. In [11], a unified approach for steady-state and tracking analysis of LMS, NLMS, and some adaptive filters with the nonlinearity property in the error is presented. The tracking analysis of the family of Affine Projection Algorithms (APAs) was presented in [12]. Their approach was based on energy-conservation relation which was originally derived in $[13,14]$. The tracking performance analysis of LMS, NLMS, APA, and RLS based on energy conservation arguments can be found in [3], but the analysis of the mentioned algorithms has been presented separately. Also, the transient and steady-state analysis of data-reusing adaptive algorithms in the stationary environment were presented in [15] based on the weighted energy relation.

In contrast to full update adaptive algorithms, the convergence analysis of adaptive filters with selective partial updates (SPU) in nonstationary environments has not been widely studied. Many contributions focus on a particular algorithm and also on stationary environment. For example in [16], the convergence analysis of the N-Max NLMS 
( $N$ is the number of filter coefficients to update) for zero mean independent Gaussian input signal and for $N=1$ is presented. In [17], the theoretical mean square performance of the SPU-NLMS algorithms was studied with the same assumption in [16]. The results in [18] present mean square convergence analysis of the SPU-NLMS for the case of white input signals. The more general performance analysis for the family of SPU-NLMS algorithms in the stationary environment can be found in $[19,20]$. The steady-state MSE analysis of SPU-NLMS in [19] was based on transient analysis. Also this paper has not presented the theoretical performance of SPU-APA. In [21], the tracking performance of some SPU adaptive filter algorithms was studied. But the analysis was presented for the white Gaussian input signal.

What we propose here is a general formalism for tracking performance analysis of the family of SPU-NLMS and SPU affine projection algorithms. Based on this, the performance of Max-NLMS [22], N-Max NLMS [16, 23], the variants of the selective partial update normalized least mean square (SPU-NLMS) [17, 18, 24], and SPU-APA [17] can be studied in nonstationary environment. The strategy of our analysis is based on energy conservation arguments and does not need to assume the Gaussian or white distribution for the regressors [25].

This paper is organized as follows. In the next section we introduce a generic update equation for the family SPUNLMS algorithms. In the next section, the general mean square performance analysis in nonstationary environment is presented. We conclude the paper by showing a comprehensive set of simulations supporting the validity of our results.

Throughout the paper, the following notations are used:

$\|\cdot\|^{2}$ : squared Euclidean norm of a vector.

$(\cdot)^{T}$ : transpose of a vector or a matrix,

$\operatorname{Tr}(\cdot)$ : trace of a matrix,

$E\{\cdot\}$ : expectation operator.

\section{Data Model and the Generic Filter Update Equation}

Figure 1 shows a typical adaptive filter setup, where $x(n)$, $d(n)$, and $e(n)$ are the input, the desired and the output error signals, respectively. Here, $\mathbf{h}(n)$ is the $M \times 1$ column vector of filter coefficients at iteration $n$.

The generic filter vector update equation at the center of our analysis is introduced as

$$
\mathbf{h}(n+1)=\mathbf{h}(n)+\mu \mathbf{C}(n) \mathbf{X}(n) \mathbf{W}(n) \mathbf{e}(n)
$$

where

$$
\mathbf{e}(n)=\mathbf{d}(n)-\mathbf{X}^{T}(n) \mathbf{h}(n)
$$

is the output error vector. The matrix $\mathbf{X}(n)$ is the $M \times P$ input signal matrix (The parameter $P$ is a positive integer (usually, but not necessarily $P \leq M)$ ),

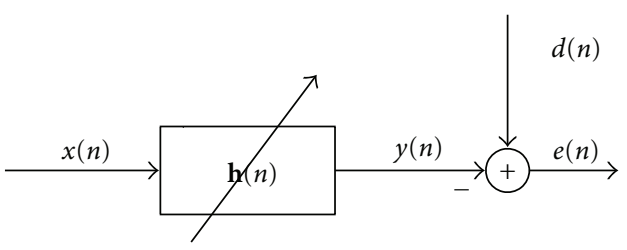

Figure 1: Prototypical adaptive filter setup.

$$
\mathbf{X}(n)=[\mathbf{x}(n), \mathbf{x}(n-1), \ldots, \mathbf{x}(n-(P-1))],
$$

where $\mathbf{x}(n)=[x(n), x(n-1), \ldots, x(n-M+1)]^{T}$ is the input signal vector, and $\mathbf{d}(n)$ is a $P \times 1$ vector of desired signal

$$
\mathbf{d}(n)=[d(n), d(n-1), \ldots, d(n-(P-1))]^{T} .
$$

The desired signal is assumed to be generated from the following linear model:

$$
\mathbf{d}(n)=\mathbf{X}^{T}(n) \mathbf{h}_{t}(n)+\mathbf{v}(n),
$$

where $\mathbf{v}(n)=[v(n), v(n-1), \ldots, v(n-(P-1))]^{T}$ is the measurement noise vector and assumed to be zero mean, white, Gaussian, and independent of the input signal, and $\mathbf{h}_{t}(n)$ is the unknown filter vector which is time-variant. We assume that the variation of $\mathbf{h}_{t}(n)$ is according to the random walk model $[1,2,25]$

$$
\mathbf{h}_{t}(n+1)=\mathbf{h}_{t}(n)+\mathbf{q}(n)
$$

where the sequence of $\mathbf{q}(n)$ is an independent and identically distributed sequence with autocorrelation matrix $\mathbf{Q}=$ $E\left\{\mathbf{q}(n) \mathbf{q}^{T}(n)\right\}$ and independent of the $\mathbf{x}(k)$ for all $k$ and of the $d(k)$ for $k<n$.

\section{Derivation of SPU Adaptive Filter Algorithms}

Different adaptive filter algorithms are established through the specific choices for the matrices $\mathbf{C}(n)$ and $\mathbf{W}(n)$ as well as for the parameter $P$.

3.1. The Family of SPU-NLMS Algorithms. From (1), the generic filter coefficients update equation for $P=1$ can be stated as

$$
\mathbf{h}(n+1)=\mathbf{h}(n)+\mu \mathbf{C}(n) \mathbf{x}(n) \mathbf{W}(n) e(n) .
$$

In the adaptive filter algorithms with selective partial updates, the $M \times 1$ vector of filter coefficients is partitioned into $K$ blocks each of length $L$ and in each iteration a subset of these blocks is updated. For this family of adaptive filters, the matrices $\mathbf{C}(n)$ and $\mathbf{W}(n)$ can be obtained from Table 1, where the $\mathbf{A}(n)$ matrix is the $M \times M$ diagonal matrix with the $\mathbf{1}$ and $\mathbf{0}$ blocks each of length $L$ on the diagonal and the positions of 1's on the diagonal determine which coefficients should be updated in each iteration. In Table 1, the parameter $L$ is the length of the block, $K$ is the number of blocks $(K=(M / L)$ and is an integer $)$ and $N$ is the number of blocks to update. Through the specific choices for $L, N$, 
TABLE 1: Family of adaptive filters with selective partial updates.

\begin{tabular}{lcccccc}
\hline Algorithm & $P$ & $L$ & $K$ & $N$ & $\mathbf{C}(n)$ & $\mathbf{W}(n)$ \\
\hline Max-NLMS [22] & 1 & 1 & $M$ & 1 & $\mathbf{A}(n)$ & $\frac{1}{\|\mathbf{A}(n) \mathbf{x}(n)\|^{2}}$ \\
N-Max NLMS [16, 23] & 1 & 1 & $M$ & $N \leq M$ & $\mathbf{A}(n)$ & $\frac{1}{\|\mathbf{x}(n)\|^{2}}$ \\
SPU-NLMS [24] & 1 & $L$ & $M / L$ & $N \leq K$ & $\mathbf{A}(n)$ & $\frac{1}{\|\mathbf{x}(n)\|^{2}}$ \\
SPU-NLMS [17, 18] & 1 & 1 & $M$ & $N \leq M$ & $\mathbf{A}(n)$ & $\frac{1}{\|\mathbf{A}(n) \mathbf{X}(n)\|^{2}}$ \\
SPU-NLMS [17] & 1 & $L$ & $M / L$ & 1 & $\mathbf{A}(n)$ & $\frac{1}{\|\mathbf{A}(n) \mathbf{X}(n)\|^{2}}$ \\
SPU-NLMS [17] & 1 & $L$ & $M / L$ & $N \leq K$ & $\mathbf{A}(n)$ & $\frac{1}{\|\mathbf{A}(n) \mathbf{x}(n)\|^{2}}$ \\
SPU-APA [17] & $P \leq M$ & $L$ & $M / L$ & $N \leq K$ & $\mathbf{A}(n)$ & $\left(\mathbf{X}^{T}(n) \mathbf{A}(n) \mathbf{X}(n)\right)^{-1}$ \\
\hline
\end{tabular}

the matrices $\mathbf{C}(n)$ and $\mathbf{W}(n)$, different SPU-NLMS adaptive filter algorithms are established.

By partitioning the regressor vector $\mathbf{x}(n)$ into $K$ blocks each of length $L$ as

$$
\mathbf{x}(n)=\left[\mathbf{x}_{1}^{T}(n), \mathbf{x}_{2}^{T}(n), \ldots, \mathbf{x}_{K}^{T}(n)\right]^{T},
$$

the positions of 1 blocks ( $N$ blocks and $N \leq K$ ) on the diagonal of $\mathbf{A}(n)$ matrix for each iteration in the family of SPU-NLMS adaptive algorithms are determined by the following procedure:

(1) the $\left\|\mathbf{x}_{i}(n)\right\|^{2}$ values are sorted for $1 \leq i \leq K$;

(2) the $i$ values that determine the positions of 1 blocks correspond to the $N$ largest values of $\left\|\mathbf{x}_{i}(n)\right\|^{2}$.

3.2. The SPU-APA. The filter vector update equation for SPU-APA is given by [17]

$$
\mathbf{h}_{F}(n+1)=\mathbf{h}_{F}(n)+\mu \mathbf{X}_{F}(n)\left(\mathbf{X}_{F}^{T}(n) \mathbf{X}_{F}(n)\right)^{-1} \mathbf{e}(n),
$$

where $F=\left\{j_{1}, j_{2}, \ldots, j_{N}\right\}$ denote the indices of the $N$ blocks out of $K$ blocks that should be updated at every adaptation, and

$$
\mathbf{X}_{F}(n)=\left[\mathbf{X}_{j_{1}}^{T}(n), \mathbf{X}_{j_{2}}^{T}(n), \ldots, \mathbf{X}_{j_{N}}^{T}(n)\right]^{T}
$$

is the $N L \times P$ matrix and

$$
\mathbf{X}_{i}(n)=\left[\mathbf{x}_{i}(n), \mathbf{x}_{i}(n-1), \ldots, \mathbf{x}_{i}(n-(P-1))\right]
$$

is the $L \times P$ matrix. The indices of $F$ are obtained by the following procedure:

(1) compute the following values for $1 \leq i \leq K$

$$
\operatorname{Tr}\left(\mathbf{X}_{i}^{T}(n) \mathbf{X}_{i}(n)\right) ;
$$

(2) the indices of $F$ are correspond to $N$ largest values of (12).
From (9), the SPU-PRA can also be established when the adaptation of the filter coefficients is performed only once every $P$ iterations. Equation (9) can be represented in the form of full update equation as

$$
\mathbf{h}(n+1)=\mathbf{h}(n)+\mu \mathbf{A}(n) \mathbf{X}(n)\left(\mathbf{X}^{T}(n) \mathbf{A}(n) \mathbf{X}(n)\right)^{-1} \mathbf{e}(n),
$$

where the $\mathbf{A}(n)$ matrix is the $M \times M$ diagonal matrix with the $\mathbf{1}$ and $\mathbf{0}$ blocks each of length $L$ on the diagonal and the positions of 1's on the diagonal determine which coefficients should be updated in each iteration. The positions of 1 blocks ( $N$ blocks and $N \leq K$ ) on the diagonal of $\mathbf{A}(n)$ matrix for each iteration in the SPU-APA is determined by the indices of $F$. Table 1 summarizes the parameters selection for the establishment of SPU-APA.

\section{Tracking Performance Analysis of the Family of SPU-NLMS and SPU-APA}

The steady-state mean square error (MSE) performance of adaptive filter algorithms can be evaluated from (14):

$$
\text { MSE }=\lim _{n \rightarrow \infty} E\left\{e^{2}(n)\right\} .
$$

In this section, we apply the energy conservation arguments approach to find the steady-state MSE of the family of SPUNLMS and SPU-AP adaptive filter algorithms. By defining the weight error vector as

$$
\widetilde{\mathbf{h}}(n)=\mathbf{h}_{t}(n)-\mathbf{h}(n),
$$

equation (1) can be stated as

$$
\begin{aligned}
\mathbf{h}_{t}(n+1)-\mathbf{h}(n+1)= & \mathbf{h}_{t}(n+1)-\mathbf{h}(n) \\
& -\mu \mathbf{C}(n) \mathbf{X}(n) \mathbf{W}(n) \mathbf{e}(n) .
\end{aligned}
$$

Substituting (6) into (16) yields

$$
\begin{aligned}
\mathbf{h}_{t}(n+1)-\mathbf{h}(n+1)= & \mathbf{h}_{t}(n)-\mathbf{h}(n)+\mathbf{q}(n) \\
& -\mu \mathbf{C}(n) \mathbf{X}(n) \mathbf{W}(n) \mathbf{e}(n) .
\end{aligned}
$$


Therefore, (17) can be written as

$$
\tilde{\mathbf{h}}(n+1)=\tilde{\mathbf{h}}(n)+\mathbf{q}(n)-\mu \mathbf{C}(n) \mathbf{X}(n) \mathbf{W}(n) \mathbf{e}(n) .
$$

By multiplying both sides of (18) from the left by $\mathbf{X}^{T}(n)$, we obtain

$$
\mathbf{e}_{p}(n)=\mathbf{e}_{a}(n)-\mu \mathbf{X}^{T}(n) \mathbf{C}(n) \mathbf{X}(n) \mathbf{W}(n) \mathbf{e}(n),
$$

where $\mathbf{e}_{a}(n)$ and $\mathbf{e}_{p}(n)$ are a priori and posteriori error vectors which are defined as

$$
\begin{aligned}
\mathbf{e}_{a}(n) & =\mathbf{X}^{T}(n)\left(\mathbf{h}_{t}(n+1)-\mathbf{h}(n)\right) \\
& =\mathbf{X}^{T}(n)\left(\mathbf{h}_{t}(n)+\mathbf{q}(n)-\mathbf{h}(n)\right) \\
& =\mathbf{X}^{T}(n)(\tilde{\mathbf{h}}(n)+\mathbf{q}(n)) \\
\mathbf{e}_{p}(n) & =\mathbf{X}^{T}(n)\left(\mathbf{h}_{t}(n+1)-\mathbf{h}(n+1)\right) \\
& =\mathbf{X}^{T}(n) \tilde{\mathbf{h}}(n+1)
\end{aligned}
$$

Finding $\mathbf{e}(n)$ from (19) and substitute it into (18), the following equality will be established:

$$
\begin{aligned}
\tilde{\mathbf{h}}(n+1) & +(\mathbf{C}(n) \mathbf{X}(n) \mathbf{W}(n))\left(\mathbf{X}^{T}(n) \mathbf{C}(n) \mathbf{X}(n) \mathbf{W}(n)\right)^{-1} \mathbf{e}_{a}(n) \\
= & \tilde{\mathbf{h}}(n)+\mathbf{q}(n)+(\mathbf{C}(n) \mathbf{X}(n) \mathbf{W}(n)) \\
& \times\left(\mathbf{X}^{T}(n) \mathbf{C}(n) \mathbf{X}(n) \mathbf{W}(n)\right)^{-1} \mathbf{e}_{p}(n) .
\end{aligned}
$$

Taking the Euclidean norm and then expectation from both sides of (21) and using the random walk model (6), we obtain after some calculations, that in the nonstationary environment the following energy equality holds:

$$
\begin{aligned}
& E\left\{\|\tilde{\mathbf{h}}(n+1)\|^{2}\right\}+E\left\{\mathbf{e}_{a}^{T}(n) \mathbf{W}(n) \mathbf{Z}^{-1}(n) \mathbf{e}_{a}(n)\right\} \\
&= E\left\{\|\tilde{\mathbf{h}}(n)\|^{2}\right\}+E\left\{\|\mathbf{q}(n)\|^{2}\right\} \\
&+E\left\{\mathbf{e}_{p}^{T}(n) \mathbf{W}(n) \mathbf{Z}^{-1}(n) \mathbf{e}_{p}(n)\right\},
\end{aligned}
$$

where $\mathbf{Z}(n)=\mathbf{X}^{T}(n) \mathbf{C}(n) \mathbf{X}(n) \mathbf{W}(n)$. Using the following steady-state condition, $E\left\{\|\tilde{\mathbf{h}}(n+1)\|^{2}\right\}=E\left\{\|\tilde{\mathbf{h}}(n)\|^{2}\right\}$, yields

$$
\begin{aligned}
& E\left\{\mathbf{e}_{a}^{T}(n) \mathbf{W}(n) \mathbf{Z}^{-1}(n) \mathbf{e}_{a}(n)\right\} \\
&=E\left\{\|\mathbf{q}(n)\|^{2}\right\}+E\left\{\mathbf{e}_{p}^{T}(n) \mathbf{W}(n) \mathbf{Z}^{-1}(n) \mathbf{e}_{p}(n)\right\} .
\end{aligned}
$$

Focusing on the second term of the right-hand side (RHS) of (23) and using (19), we obtain

$$
\begin{aligned}
E\left\{\mathbf{e}_{p}^{T}(n)\right. & \left.\mathbf{W}(n) \mathbf{Z}^{-1}(n) \mathbf{e}_{p}(n)\right\} \\
= & E\left\{\mathbf{e}_{a}^{T}(n) \mathbf{W}(n) \mathbf{Z}^{-1}(n) \mathbf{e}_{a}(n)\right\} \\
& -\mu E\left\{\mathbf{e}_{a}^{T}(n) \mathbf{W}(n) \mathbf{e}(n)\right\} \\
& -\mu E\left\{\mathbf{e}^{T}(n) \mathbf{Z}^{T}(n) \mathbf{W}(n) \mathbf{Z}^{-1}(n) \mathbf{e}_{a}(n)\right\} \\
& +\mu^{2} E\left\{\mathbf{e}^{T}(n) \mathbf{Z}^{T}(n) \mathbf{W}(n) \mathbf{Z}^{-1}(n) \mathbf{e}(n)\right\} .
\end{aligned}
$$

By substituting (24) into the second term of RHS of (23) and eliminating the equal terms from both sides, we have

$$
\begin{aligned}
-\mu E\left\{\mathbf{e}_{a}^{T}(n) \mathbf{W}(n) \mathbf{e}(n)\right\} \\
-\mu E\left\{\mathbf{e}^{T}(n) \mathbf{Z}^{T}(n) \mathbf{W}(n) \mathbf{Z}^{-1}(n) \mathbf{e}_{a}(n)\right\} \\
+\mu^{2} E\left\{\mathbf{e}^{T}(n) \mathbf{Z}^{T}(n) \mathbf{W}(n) \mathbf{Z}^{-1}(n) \mathbf{e}(n)\right\} \\
+E\left\{\|\mathbf{q}(n)\|^{2}\right\}=0 .
\end{aligned}
$$

From (2) and (5), the relation between the output estimation error and a priori estimation error vectors is given by

$$
\mathbf{e}(n)=\mathbf{e}_{a}(n)+\mathbf{v}(n) .
$$

Using (26), we obtain

$$
\begin{aligned}
-\mu E\left\{\mathbf{e}_{a}^{T}(n) \mathbf{W}(n) \mathbf{e}_{a}(n)\right\} \\
-\mu E\left\{\mathbf{e}_{a}^{T}(n) \mathbf{Z}^{T}(n) \mathbf{W}(n) \mathbf{Z}^{-1}(n) \mathbf{e}_{a}(n)\right\} \\
+\mu^{2} E\left\{\mathbf{e}_{a}^{T}(n) \mathbf{Z}^{T}(n) \mathbf{W}(n) \mathbf{e}_{a}(n)\right\} \\
+\mu^{2} E\left\{\mathbf{v}^{T}(n) \mathbf{Z}^{T}(n) \mathbf{W}(n) \mathbf{v}(n)\right\}+\operatorname{Tr}(\mathbf{Q})=0 .
\end{aligned}
$$

The steady-state excess MSE (EMSE) is defined as

$$
\mathrm{EMSE}=\lim _{n \rightarrow \infty} E\left\{e_{a}^{2}(n)\right\},
$$

where $e_{a}(n)$ is the a priori error signal. To obtain the steadystate EMSE, we need the following assumption from [12]. At steady-state the input signal and therefore $\mathbf{Z}(n)$ and $\mathbf{W}(n)$ are statistically independent of $\mathbf{e}_{a}(n)$ and moreover $E\left\{\mathbf{e}_{a}(n) \mathbf{e}_{a}^{T}(n)\right\}=E\left\{e_{a}^{2}(n)\right\} \cdot \mathbf{S}$ where $\mathbf{S} \approx \mathbf{I}_{P \times P}$ for small $\mu$ and $\mathbf{S} \approx\left(\mathbf{1} \cdot \mathbf{1}^{T}\right)$ for large $\mu$ where $\mathbf{1}^{T}=[1,0, \ldots, 0]_{1 \times P}$.

Based on this, we analyze four parts of (27),

Part I:

$$
E\left\{\mathbf{e}_{a}^{T}(n) \mathbf{W}(n) \mathbf{e}_{a}(n)\right\}=E\left\{e_{a}^{2}(n)\right\} \operatorname{Tr}(\mathbf{S} E\{\mathbf{W}(n)\}) .
$$

Part II:

$$
\begin{aligned}
& E\left\{\mathbf{e}_{a}^{T}(n) \mathbf{Z}^{T}(n) \mathbf{W}(n) \mathbf{Z}^{-1}(n) \mathbf{e}_{a}(n)\right\} \\
&=E\left\{e_{a}^{2}(n)\right\} \operatorname{Tr}\left(\mathbf{S} E\left\{\mathbf{Z}^{T}(n) \mathbf{W}(n) \mathbf{Z}^{-1}(n)\right\}\right) .
\end{aligned}
$$


Part III:

$$
\begin{aligned}
E\left\{\mathbf{e}_{a}^{T}(n) \mathbf{Z}^{T}(n) \mathbf{W}(n) \mathbf{e}_{a}(n)\right\} & \\
= & E\left\{e_{a}^{2}(n)\right\} \operatorname{Tr}\left(\mathbf{S} E\left\{\mathbf{Z}^{T}(n) \mathbf{W}(n)\right\}\right) .
\end{aligned}
$$

$$
E\left\{\mathbf{v}^{T}(n) \mathbf{Z}^{T}(n) \mathbf{W}(n) \mathbf{v}(n)\right\}=\sigma_{v}^{2} \operatorname{Tr}\left(E\left\{\mathbf{Z}^{T}(n) \mathbf{W}(n)\right\}\right) .
$$

Therefore from (27), the EMSE is given by

$$
E\left\{e_{a}^{2}(n)\right\}=\mathrm{EMSE}=\frac{\mu \sigma_{v}^{2} \operatorname{Tr}\left(E\left\{\mathbf{Z}^{T}(n) \mathbf{W}(n)\right\}\right)+\mu^{-1} \operatorname{Tr}(\mathbf{Q})}{\operatorname{Tr}(\mathbf{S} E\{\mathbf{W}(n)\})+\operatorname{Tr}\left(\mathbf{S} E\left\{\mathbf{Z}^{T}(n) \mathbf{W}(n) \mathbf{Z}^{-1}(n)\right\}\right)-\mu \operatorname{Tr}\left(\mathbf{S} E\left\{\mathbf{Z}^{T}(n) \mathbf{W}(n)\right\}\right)} .
$$

Also from (26), the steady-state MSE can be obtained by

$$
\mathrm{MSE}=\mathrm{EMSE}+\sigma_{v}^{2} .
$$

From the general expression (33), we will be able to predict the steady-state MSE of the family of SPU-NLMS, and SPUAP adaptive filter algorithms in the nonstationary environment. Selecting $\mathbf{A}(n)=\mathbf{I}$ and the parameters selection according to Table 1, the tracking performance of NLMS and APA can also be analyzed.

\section{Simulation Results}

The theoretical results presented in this paper are confirmed by several computer simulations for a system identification setup. The unknown systems have 8 and 16, where the taps are randomly selected. The input signal $x(n)$ is a first-order autoregressive (AR) signal generated by

$$
x(n)=\rho x(n-1)+w(n)
$$

where $w(n)$ is either a zero mean white Gaussian signal or a zero mean uniformly distributed random sequence between -1 and 1 . For the Gaussian case, the value of $\rho$ is set to 0.9 , generating a highly colored Gaussian signal. For the uniform distribution case, the value of $\rho$ is set to 0.5 . The measurement noise $v(n)$ with $\sigma_{v}^{2}=10^{-3}$ is added to the noise free desired signal $d(n)=\mathbf{h}_{t}^{T}(n) \mathbf{x}(n)$. The adaptive filter and the unknown channel are assumed to have the same number of taps. In all simulations, the simulated learning curves are obtained by ensemble averaging over 200 independent trials. Also, the steady-state MSE is obtained by averaging over 500 steady-state samples from 500 independent realizations for each value of $\mu$ for a given algorithm. Also, we assume an independent and identically distributed sequence for $\mathbf{q}(n)$ with autocorrelation matrix $\mathbf{Q}=\sigma_{q}^{2} \cdot \mathbf{I}$ where $\sigma_{q}^{2}=0.0025 \sigma_{v}^{2}$.

Figures 2-5 show the steady-state MSE of the $N$-Max NLMS adaptive algorithm for $M=8$, and different values for $N$ as a function of step size in a nonstationary environment. The step size changes in the stability bound for both colored Gaussian and uniform distribution input signals. Figure 2 shows the results for $N=4$, and for diffrent input signals. The theoretical results are from (33). As we can see, the theoretical values are in good agreement with simulation results. This agreement is better for uniform input signal. Figure 3 presents the results for $N=5$. Again, the agreement is good, specially for uniform input signal. In Figures 4 and

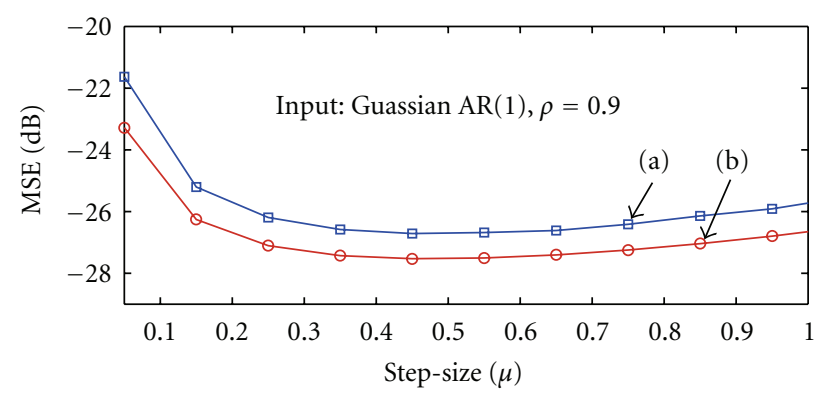

$\square$ (a) $N$-max NLMS, $K=8, N=4$, simulation

$\bigcirc$ (b) $N$-max NLMS, $K=8, N=4$, theory

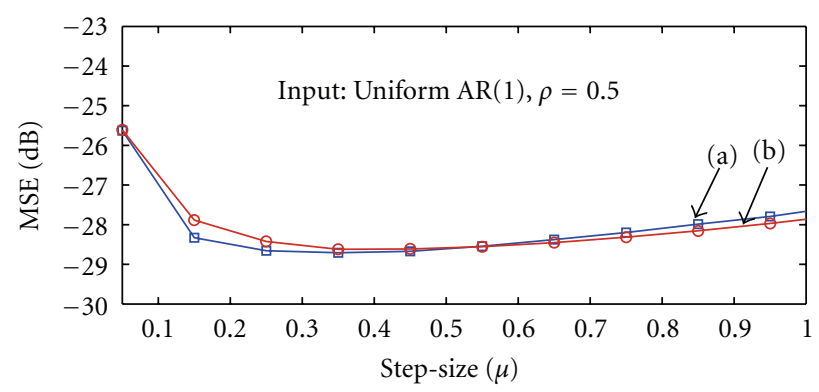

$\square$ (a) $N$-max NLMS, $K=8, N=4$, simulation

$\bigcirc$ (b) $N$-max NLMS, $K=8, N=4$, theory

Figure 2: Steady-state MSE of $N$-Max NLMS with $M=8$ and $N=$ 4 as a function of the step size in nonstationary environment for different input signals.

5, we presented the results for $N=6$, and $N=7$ respectively. This figure shows that the derived theoretical expression is suitable to predict the steady-state MSE of $N$-Max NLMS adaptive filter algorithm in nonstationary environment.

Figures 6-8 show the steady-state MSE of SPU-NLMS adaptive algorithm with $M=8$ as a function of step size in a nonstationary environment for colored Gaussian and uniform input signals. We set the number of block $(K)$ to 4 and different values for $N$ is chosen in simulations. Figure 6 presents the results for $N=2$ and for different input signals. The good agreement between the theoretical steady-state MSE and the simulated steady-state MSE is observed. This fact can be seen in Figures 7 and 8 for $N=3$, and $N=4$ respectively. 


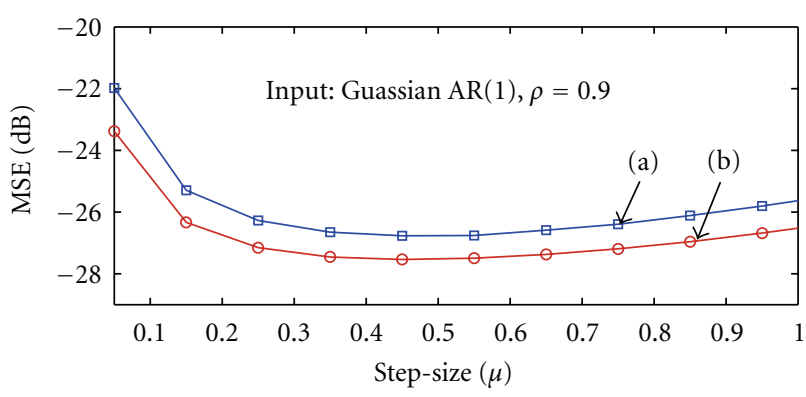

$\square$ (a) $N$-max NLMS, $K=8, N=5$, simulation

(b) $N$-max NLMS, $K=8, N=5$, theory

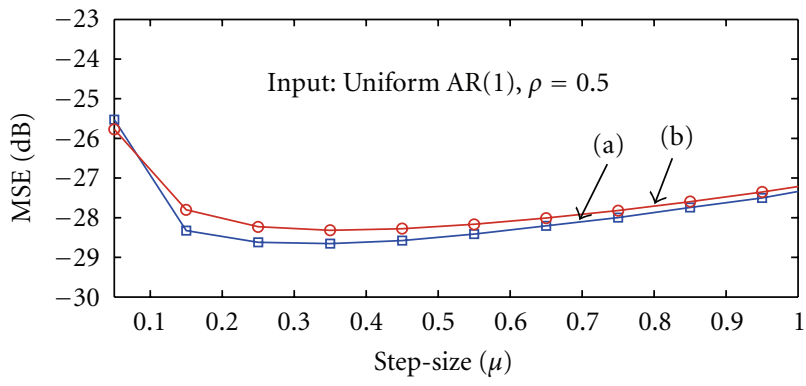

$\square$ (a) $N$-max NLMS, $K=8, N=5$, simulation

(b) $N$-max NLMS, $K=8, N=5$, theory

FIgURE 3: Steady-state MSE of $N$-Max NLMS with $M=8$ and $N=$ 5 as a function of the step size in nonstationary environment for different input signals.

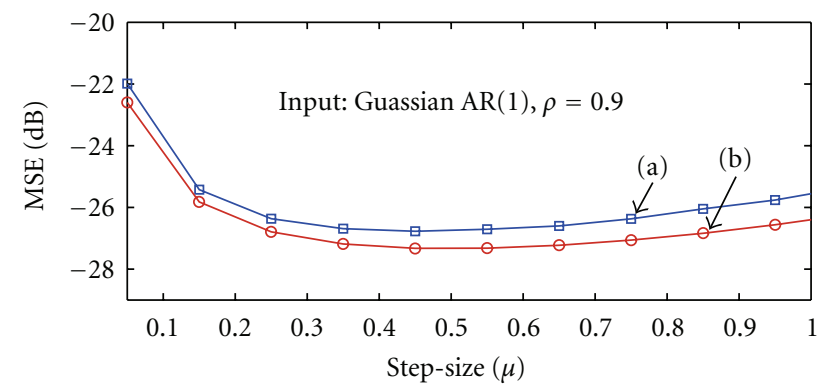

$\square$ (a) $N$-max NLMS, $K=8, N=6$, simulation

(b) $N$-max NLMS, $K=8, N=6$, theory

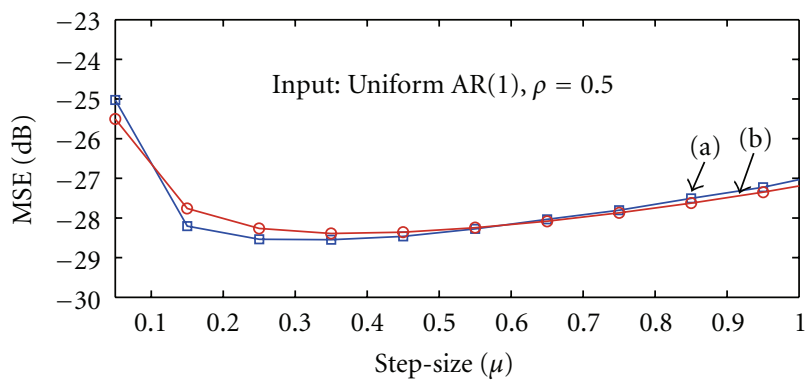

$\square$ (a) $N$-max NLMS, $K=8, N=6$, simulation

(b) $N$-max NLMS, $K=8, N=6$, theory

Figure 4: Steady-state MSE of $N$-Max NLMS with $M=8$ and $N=$ 6 as a function of the step size in nonstationary environment for different input signals.

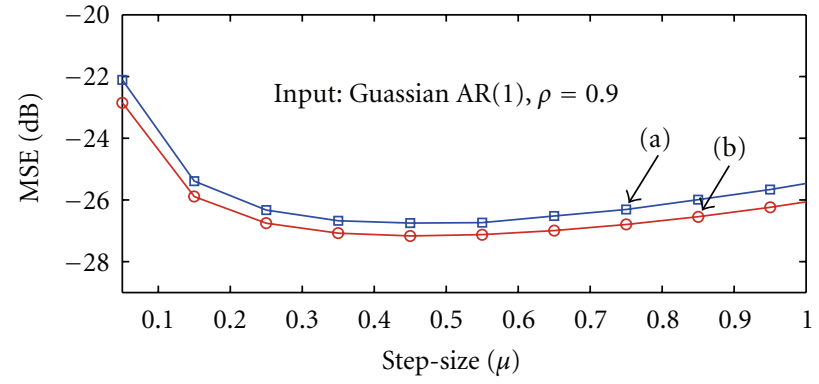

$\square$ (a) $N$-max NLMS, $K=8, N=7$, simulation

(b) $N$-max NLMS, $K=8, N=7$, theory

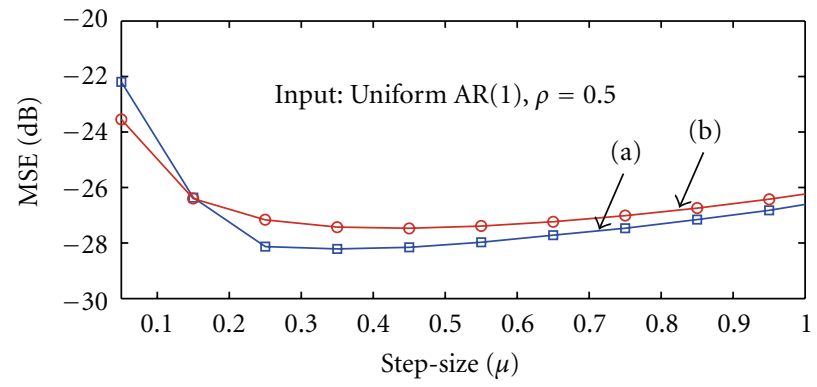

$\square$ (a) $N$-max NLMS, $K=8, N=7$, simulation

(b) $N$-max NLMS, $K=8, N=7$, theory

Figure 5: Steady-state MSE of $N$-Max NLMS with $M=8$ and $N=$ 7 as a function of the step size in nonstationary environment for different input signals.

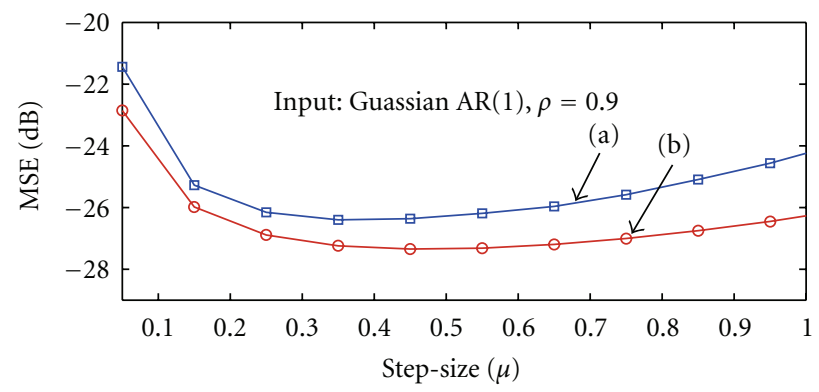

$\square$ (a) SPU-NLMS, $M=8, K=4, N=2$, simulation

(b) SPU-NLMS, $M=8, K=4, N=2$, theory

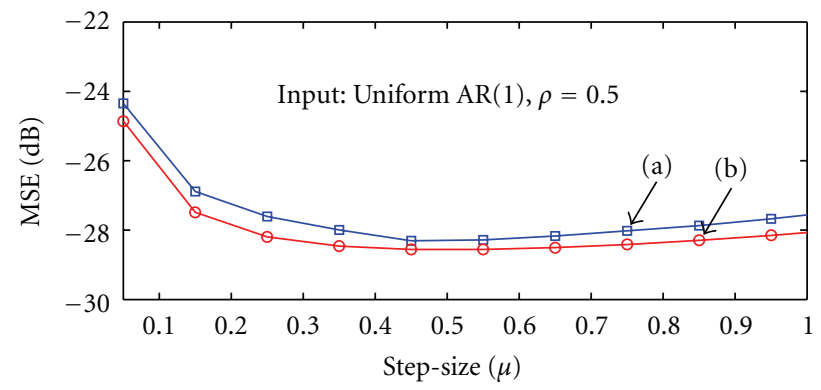

$\square$ (a) SPU-NLMS, $M=8, K=4, N=2$, simulation

$\bigcirc$ (b) SPU-NLMS, $M=8, K=4, N=2$, theory

FIgURE 6: Steady-state MSE of SPU-NLMS with $M=8, K=4$ and $N=2$ as a function of the step size in nonstationary environment for different input signals. 


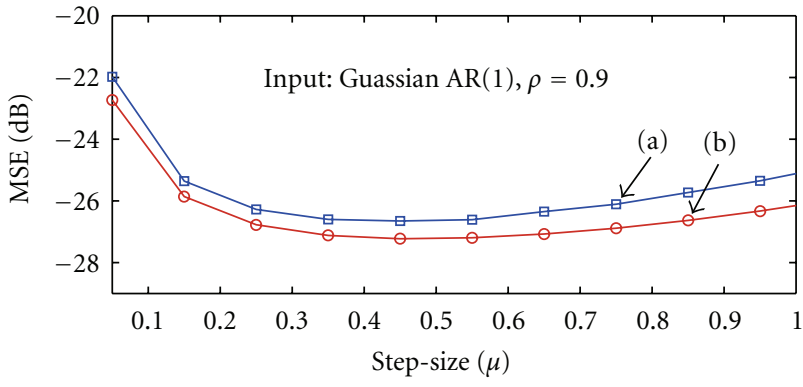

$\square$ (a) SPU-NLMS, $M=8, K=4, N=3$, simulation

(b) SPU-NLMS, $M=8, K=4, N=3$, theory

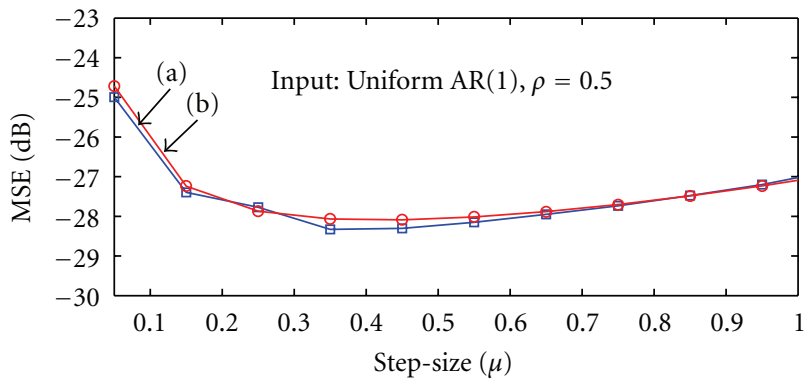

$\square$ (a) SPU-NLMS, $M=8, K=4, N=3$, simulation

(b) SPU-NLMS, $M=8, K=4, N=3$, theory

Figure 7: Steady-state MSE of SPU-NLMS with $M=8, K=4$ and $N=3$ as a function of the step size in nonstationary environment for different input signals.

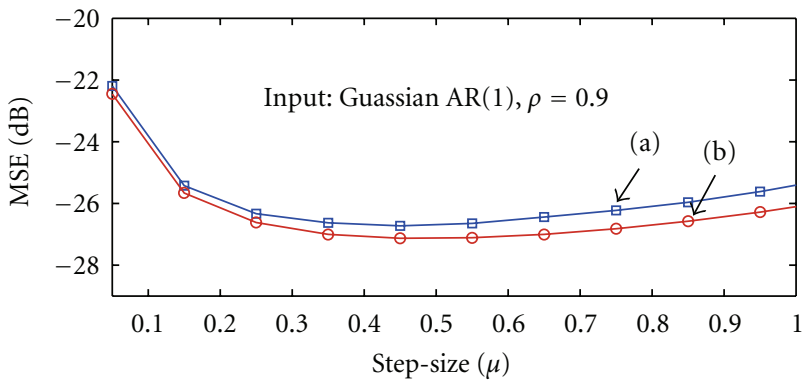

$\square$ (a) SPU-NLMS, $M=8, K=4, N=4$, simulation

$\bigcirc$ (b) SPU-NLMS, $M=8, K=4, N=4$, theory

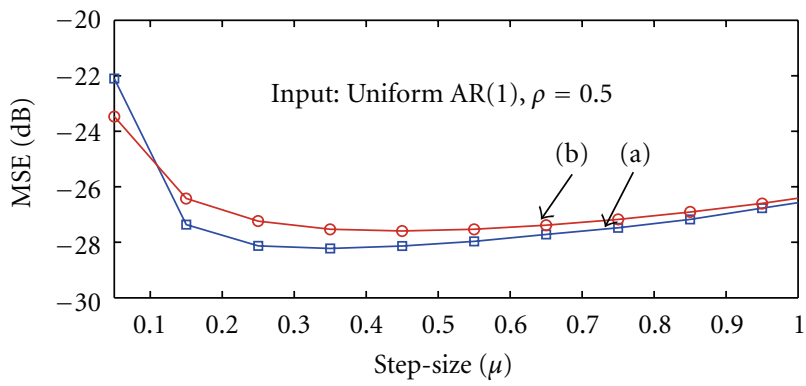

$\square$ (a) SPU-NLMS, $M=8, K=4, N=4$, simulation

(b) SPU-NLMS, $M=8, K=4, N=4$, theory

Figure 8: Steady-state MSE of SPU-NLMS with $M=8, K=4$ and $N=4$ as a function of the step size in nonstationary environment for different input signals.

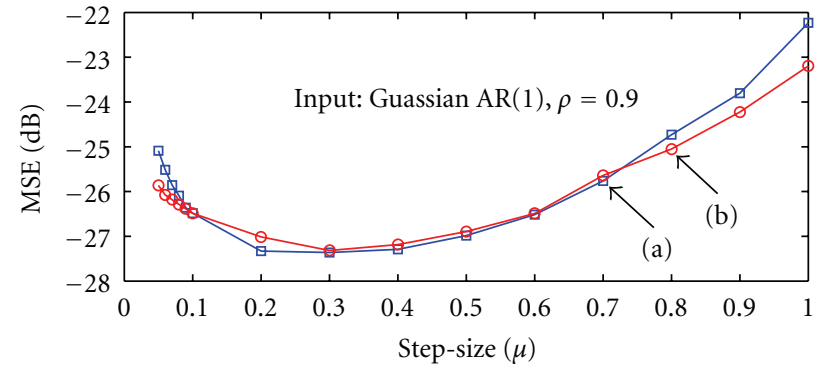

$\square$ (a) SPU-APA, $M=8, P=4, K=4, N=2$, simulation

$\bigcirc$ (b) SPU-APA, $M=8, P=4, K=4, N=2$, theory

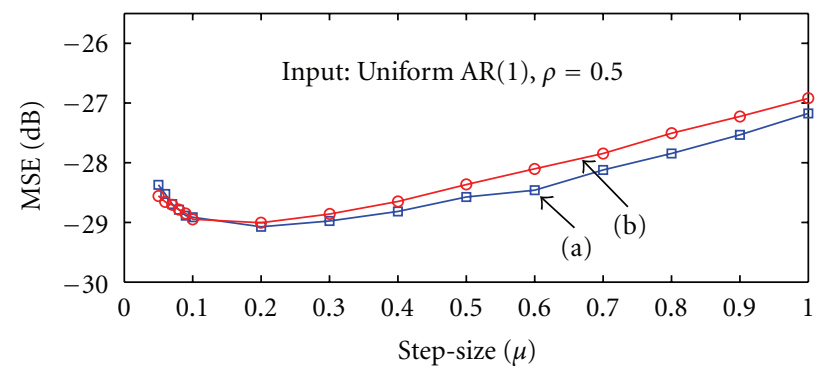

$\square$ (a) SPU-APA, $M=8, P=4, K=4, N=2$, simulation

$\checkmark$ (b) SPU-APA, $M=8, P=4, K=4, N=2$, theory

Figure 9: Steady-state MSE of SPU-APA with $M=8, P=4$, $K=4$ and $N=2$ as a function of the step size in nonstationary environment for different input signals.

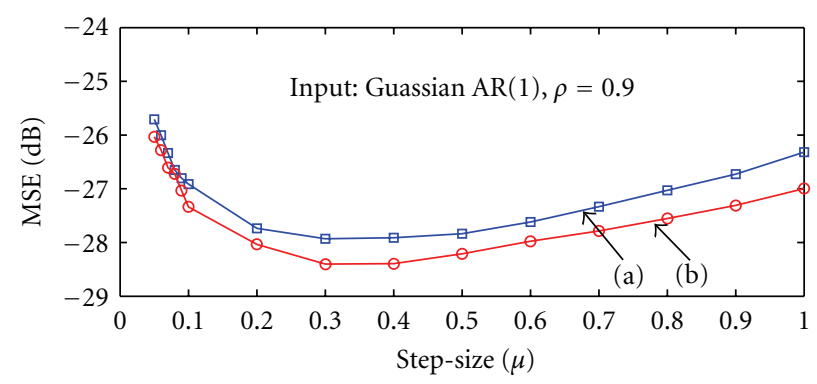

$\square$ (a) SPU-APA, $M=8, P=4, K=4, N=3$, simulation

(b) SPU-APA, $M=8, P=4, K=4, N=3$, theory

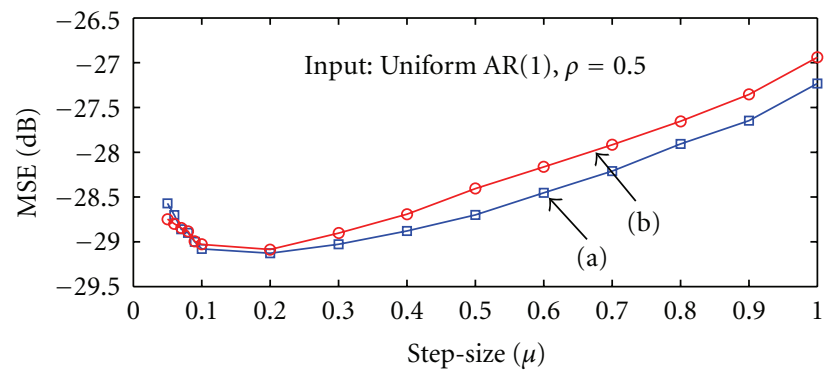

$\square$ (a) SPU-APA, $M=8, P=4, K=4, N=3$, simulation

$\bigcirc$ (b) SPU-APA, $M=8, P=4, K=4, N=3$, theory

FIgURe 10: Steady-state MSE of SPU-APA with $M=8, P=4$, $K=4$ and $N=3$ as a function of the step size in nonstationary environment for different input signals. 


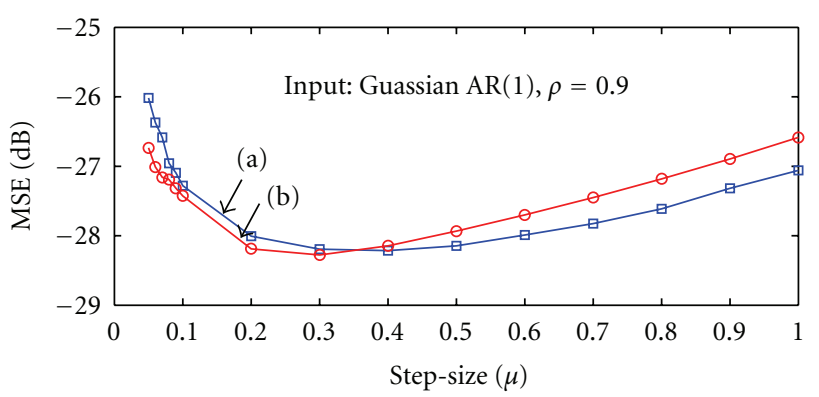

$\square$ (a) SPU-APA, $M=8, P=4, K=4, N=4$, simulation

$\bigcirc$ (b) SPU-APA, $M=8, P=4, K=4, N=4$, theory

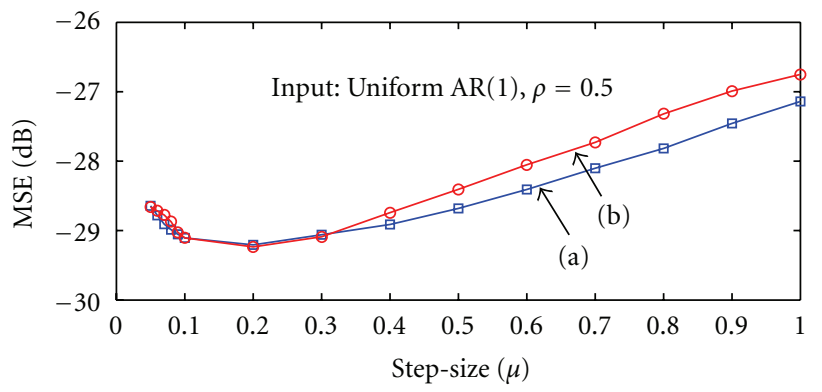

$\square$ (a) SPU-APA, $M=8, P=4, K=4, N=4$, simulation

$\bigcirc$ (b) SPU-APA, $M=8, P=4, K=4, N=4$, theory

Figure 11: Steady-state MSE of SPU-APA with $M=8, P=4$, $K=4$ and $N=4$ as a function of the step size in nonstationary environment for different input signals.

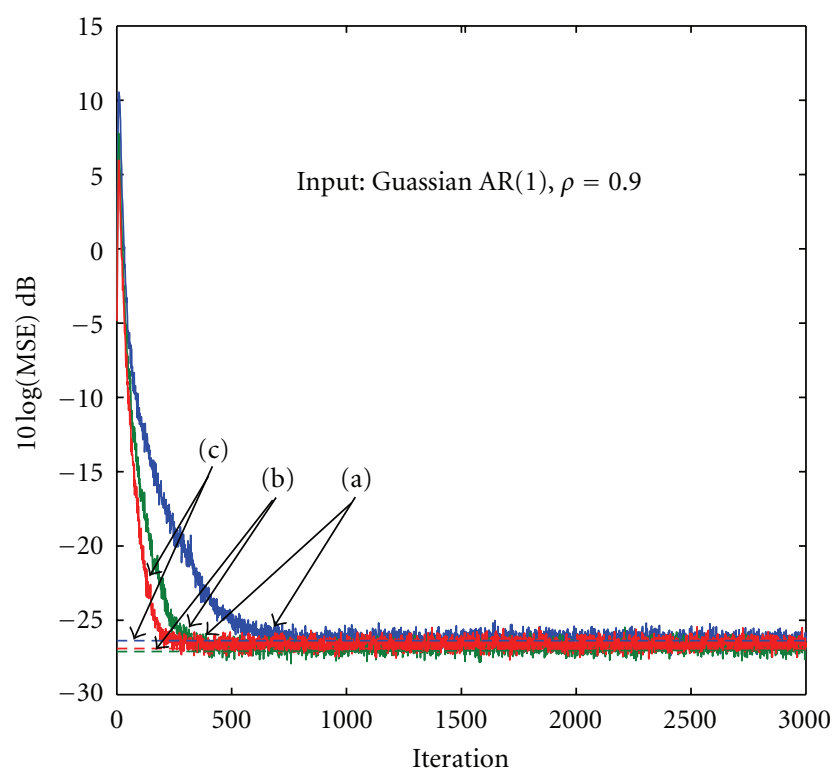

(a) $N$-max NLMS, $K=8, N=4, \mu=0.2$

(b) $N$-max NLMS, $K=8, N=4, \mu=0.4$

- (c) $N$-max NLMS, $K=8, N=4, \mu=0.6$

- - - Theoretical

FIgure 12: Learning curves of $N$-Max NLMS with $M=8$ and $N=$ 4 and different values of the step size for colored Gaussian input signal.

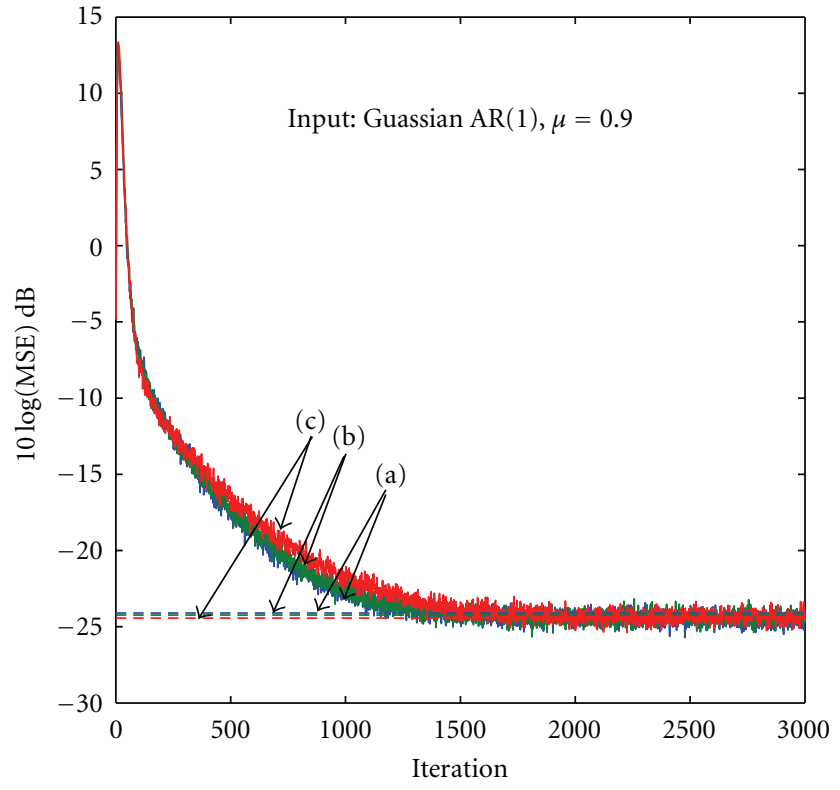

Figure 13: Learning curves of SPU-NLMS with $M=8, K=4$, and $N=2,3,4$ for colored Gaussian input signal.

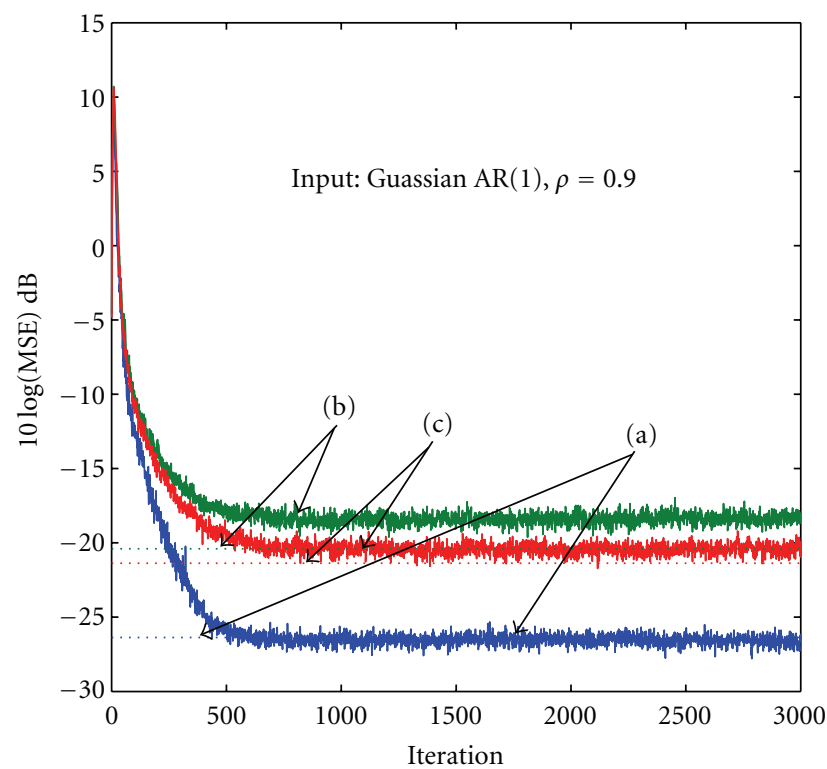

- (a) SPU-NLMS, $M=8, K=4, N=3, \mu=0.1, \sigma_{q}^{2}=0.0025 \sigma_{v}^{2}$

- (b) SPU-NLMS, $M=8, K=4, N=3, \mu=0.1, \sigma_{q}^{2}=0.025 \sigma_{v}^{2}$

(c) SPU-NLMS, $M=8, K=4, N=3, \mu=0.1, \sigma_{q}^{2}=0.0015 \sigma_{v}^{2}$

- - - Theoretical

FIgURE 14: Learning curves of SPU-NLMS with $M=8, K=4$ and $N=3$ for different degree of nonstationary and for colored Gaussian input signal. 


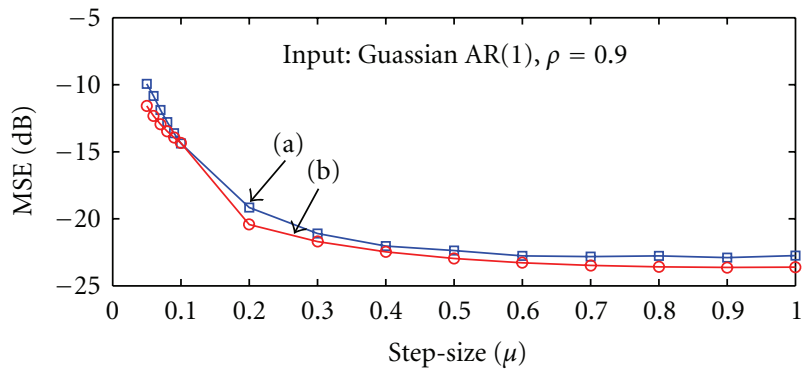

(a) SPU-NLMS, $M=16, K=4, N=2$, simulation

(b) SPU-NLMS, $M=16, K=4, N=2$, theory

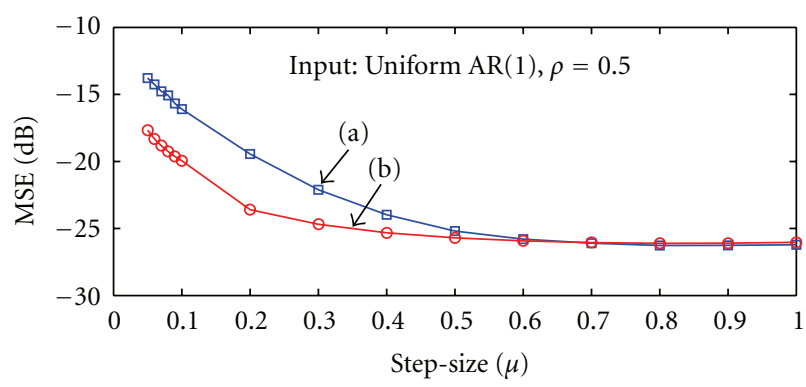

(a) SPU-NLMS, $M=16, K=4, N=2$, simulation

(b) SPU-NLMS, $M=16, K=4, N=2$, theory

FIgURE 15: Steady-state MSE of SPU-NLMS with $M=16, K=$ 4 and $N=2$ as a function of the step size in nonstationary environment for different input signals.

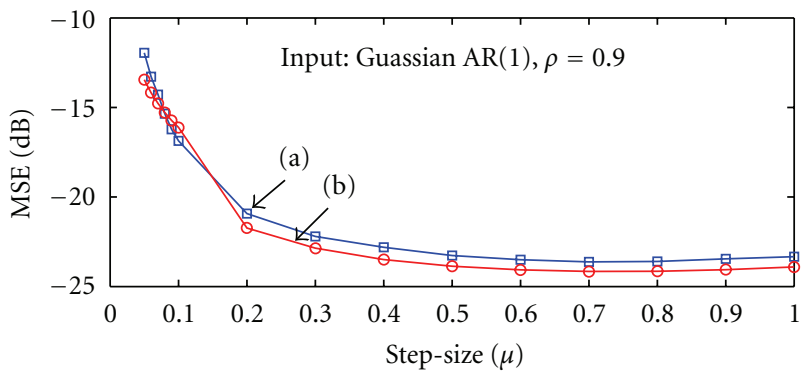

$\square$ (a) SPU-NLMS, $M=16, K=4, N=3$, simulation

(b) SPU-NLMS, $M=16, K=4, N=3$, theory

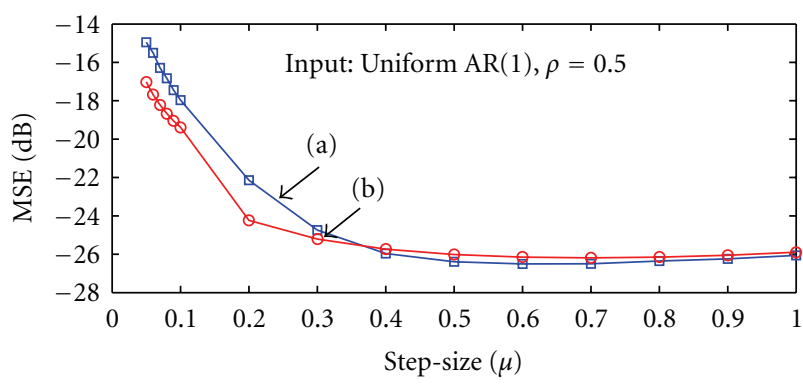

(a) SPU-NLMS, $M=16, K=4, N=3$, simulation

$\bigcirc$ (b) SPU-NLMS, $M=16, K=4, N=3$, theory

Figure 16: Steady-state MSE of SPU-NLMS with $M=16, K=$ 4 , and $N=3$ as a function of the step size in nonstationary environment for different input signals.

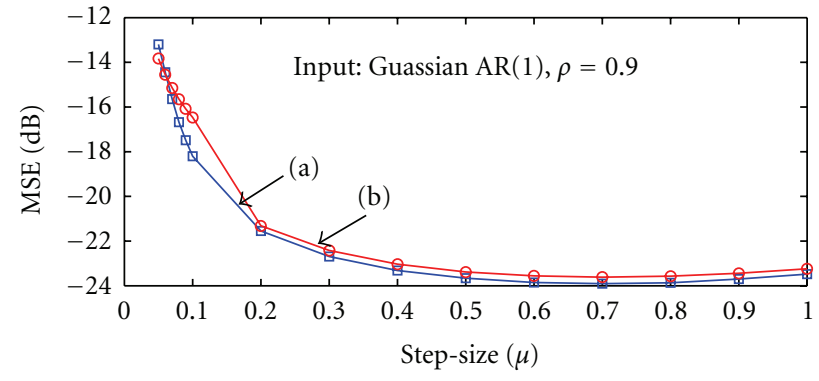

$\square$ (a) SPU-NLMS, $M=16, K=4, N=4$, simulation

(b) SPU-NLMS, $M=16, K=4, N=4$, theory

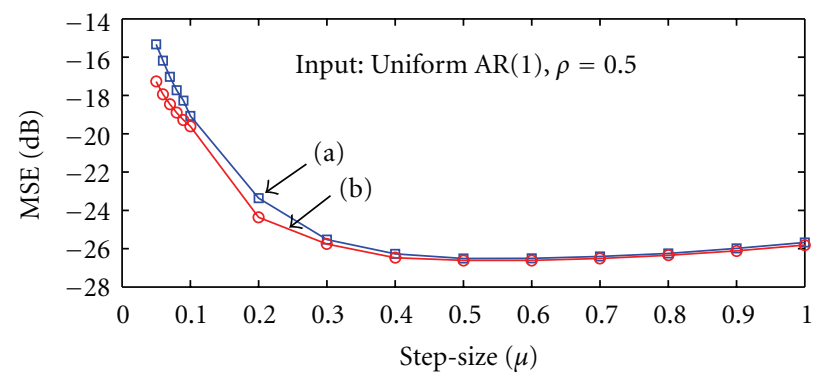

(a) SPU-NLMS, $M=16, K=4, N=4$, simulation

(b) SPU-NLMS, $M=16, K=4, N=4$, theory

Figure 17: Steady-state MSE of SPU-NLMS with $M=16, K=$ 4 , and $N=4$ as a function of the step size in nonstationary environment for different input signals.

Figures 9-11 show the steady-state MSE of SPU-APA as a function of step size for $M=8$, and different input signals. The parameters $K$, and $P$ were set to 4 , and the step size changes from 0.05 to 1 . Different values for $N$ have been used in simulations. Figure 9 shows the results for $N=2$. Simulation results show good agreement for both colored and uniform input signals. In Figure 10, we set the parameter $N$ to 3 . Again good agreement can be seen especially for uniform input signal. Finally, Figure 11 shows the results for $N=4$. As we can see, the presented theoretical relation is suitable to predict the steady-state MSE.

Figures 12-14 show the simulated learning curves of SPU adaptive filter algorithms for different parameters values and for colored Gaussian input signal. Figure 12 presents the learning curves for N-Max NLMS algorithm with $M=$ $8, N=4$ and different values for the step size. Also, the theoretical steady-state MSE was calculated based on (33) and compared with simulated steady-state MSE. As we can see the theoretical values are in good agreement with simulation results. Figure 13 shows the learning curves of SPU-NLMS algorithm with $M=8, K=4$, and $N=2,3,4$. Also, the step size was set to 0.1. Again the theoretical values of the steady-state MSE has been shown in this figure. Again good agreement is observed. In Figure 14, the learning curves of SPU-NLMS with $M=8, K=4$, and $N=3$, have been presented for different values of $\sigma_{q}^{2}$. The degree of nonstationary changes by selecting different values for $\sigma_{q}^{2}$. As we can see, for the large values of $\sigma_{q}^{2}$, the agreement between 


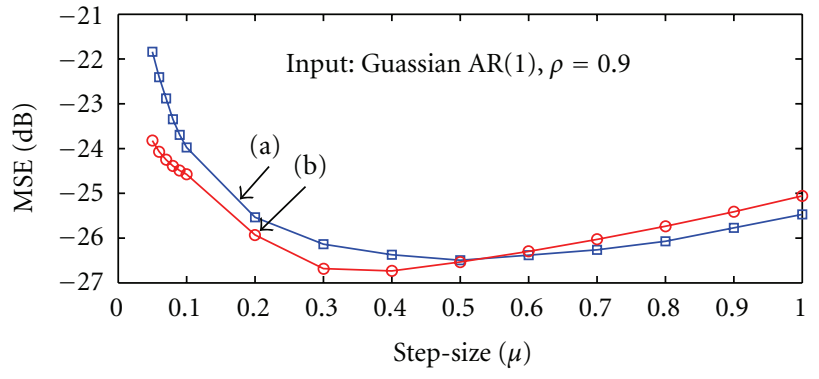

$\square$ (a) SPU-APA, $M=16, P=4, K=4, N=3$, simulation

$\checkmark$ (b) SPU-APA, $M=16, P=4, K=4, N=3$, theory

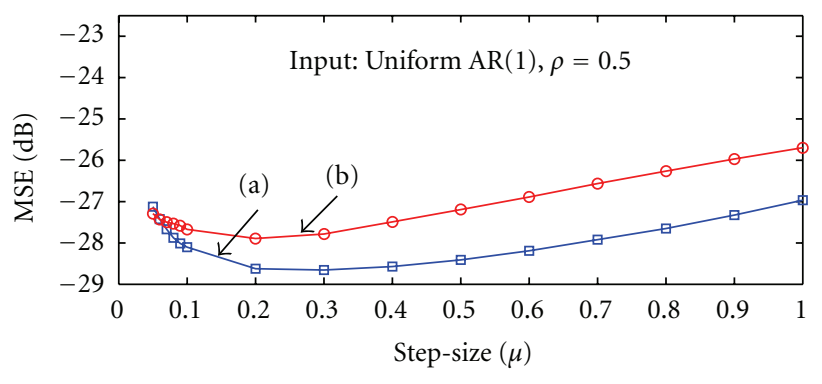

(a) SPU-APA, $M=16, P=4, K=4, N=3$, simulation

(b) SPU-APA, $M=16, P=4, K=4, N=3$, theory

FIGURE 18: Steady-state MSE of SPU-APA with $M=16, P=4, K=4$, and $N=3$ as a function of the step size in nonstationary environment for different input signals.

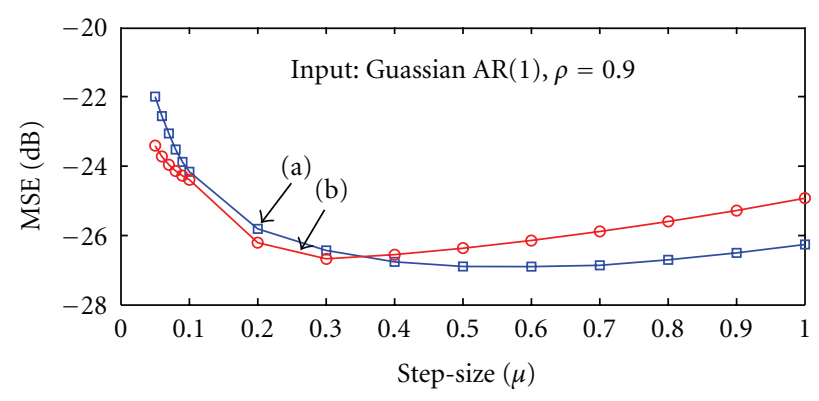

$\square$ (a) SPU-APA, $M=16, P=4, K=4, N=4$, simulation

(b) SPU-APA, $M=16, P=4, K=4, N=4$, theory

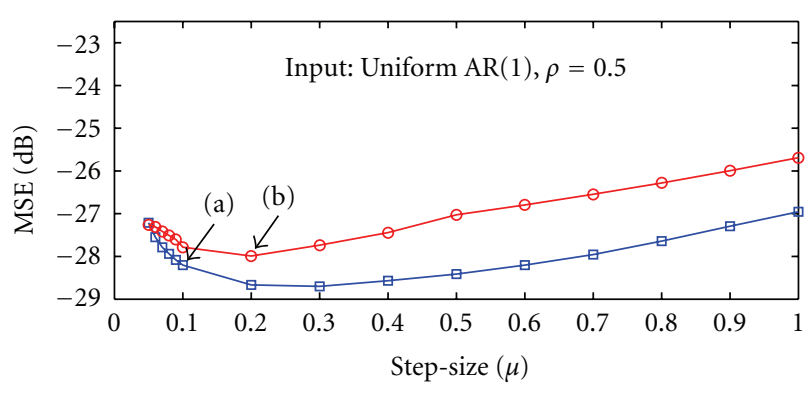

$\square$ (a) SPU-APA, $M=16, P=4, K=4, N=4$, simulation (b) SPU-APA, $M=16, P=4, K=4, N=4$, theory

FIGURE 19: Steady-state MSE of SPU-APA with $M=16, P=4, K=4$, and $N=4$ as a function of the step size in nonstationary environment for different input signals.

simulated steady-state MSE and theoretical steady-state MSE is deviated.

Figures 15-17 show the steady-state MSE of SPU-NLMS adaptive algorithm with $M=16$ as a function of step size in a nonstationary environment for colored Gaussian and uniform input signals. We set the number of blocks $(K)$ to 4 and different values for $N$ are chosen in simulations. Figure 15 presents the results for $N=2$ and for different input signals. The good agreement between the theoretical steady-state MSE and the simulated steady-state MSE is observed. In Figures 16 and 17, we presented the results for $N=3$, and 4. Simulation results show good agreement for both colored and uniform input signals.

Figures 18 and 19 show the steady-state MSE of SPUAPA as a function of step size for $M=16$, and different input signals. The parameters $K$, and $P$ were set to 4 , and the step size changes from 0.04 to 1 . Different values for $N$ have been used in simulations. Figure 18 shows the results for $N=3$. In Figure 19, the parameter $N$ was set to 4 . Again good agreement can be seen for both input signals.
The simulation results show that the agreement is deviated for $M=16$.

\section{Summary and Conclusions}

We presented a general framework for tracking performance analysis of the family of SPU-NLMS adaptive filter algorithms in nonstationary environment. Using the general expression and for the parameter values in Table 1, the mean square performances of Max-NLMS, N-Max NLMS, the various types of SPU-NLMS, and SPU-APA can be analyzed in a unified way. We demonstrated the usefulness of the presented analysis through several simulation results.

\section{References}

[1] B. Widrow and S. D. Stearns, Adaptive Signal Processing, Prentice Hall, Englewood Cliffs, NJ, USA, 1985.

[2] S. Haykin, Adaptive Filter Theory, Prentice Hall, Englewood Cliffs, NJ, USA, 4th edition, 2002. 
[3] A. H. Sayed, Adaptive Filters, John Wiley \& Sons, New York, NY, USA, 2008.

[4] B. Widrow, J. M. McCool, M. G. Larimore, and C. R. Johnson Jr., "Stationary and nonstationry learning characteristics of the LMS adaptive filter," Proceedings of the IEEE, vol. 64, no. 8, pp. 1151-1162, 1976.

[5] N. J. Bershad, F. A. Reed, P. L. Feintuch, and B. Fisher, "Tracking charcteristics of the LMS adaptive line enhancer: response to a linear chrip signal in noise," IEEE Transactions on Acoustics, Speech, and Signal Processing, vol. 28, no. 5, pp. 504-516, 1980.

[6] S. Marcos and O. Macchi, "Tracking capability of the least mean square algorithm: application to an asynchronous echo canceller," IEEE Transactions on Acoustics, Speech, and Signal Processing, vol. 35, no. 11, pp. 1570-1578, 1987.

[7] E. Eweda, "Analysis and design of a signed regressor LMS algorithm for stationary and nonstationary adaptive filtering with correlated Gaussian data," IEEE Transactions on Circuits and Systems, vol. 37, no. 11, pp. 1367-1374, 1990.

[8] E. Eweda, "Optimum step size of sign algorithm for nonstationary adaptive filtering," IEEE Transactions on Acoustics, Speech, and Signal Processing, vol. 38, no. 11, pp. 1897-1901, 1990.

[9] E. Eweda, "Comparison of RLS, LMS, and sign algorithms for tracking randomly time-varying channels," IEEE Transactions on Signal Processing, vol. 42, no. 11, pp. 2937-2944, 1994.

[10] N. R. Yousef and A. H. Sayed, "Steady-state and tracking analyses of the sign algorithm without the explicit use of the independence assumption," IEEE Signal Processing Letters, vol. 7, no. 11, pp. 307-309, 2000.

[11] N. R. Yousef and A. H. Sayed, "A unified approach to the steady-state and tracking analyses of adaptive filters," IEEE Transactions on Signal Processing, vol. 49, no. 2, pp. 314-324, 2001.

[12] H.-C. Shin and A. H. Sayed, "Mean-square performance of a family of affine projection algorithms," IEEE Transactions on Signal Processing, vol. 52, no. 1, pp. 90-102, 2004.

[13] A. H. Sayed and M. Rupp, "A time-domain feedback analysis of adaptive algorithms via the small gain theorem," in Advanced Signal Processing Algorithms, vol. 2563 of Proceedings of SPIE, San Diego, Calif, USA, 1995.

[14] M. Rupp and A. H. Sayed, "A time-domain feedback analysis of filteredor adaptive gradient algorithms," IEEE Transactions on Signal Processing, vol. 44, no. 6, pp. 1428-1439, 1996.

[15] H.-C. Shin, W.-J. Song, and A. H. Sayed, "Mean-square performance of data-reusing adaptive algorithms," IEEE Signal Processing Letters, vol. 12, no. 12, pp. 851-854, 2005.

[16] T. Aboulnasr and K. Mayyas, "Complexity reduction of the NLMS algorithm via selective coefficient update," IEEE Transactions on Signal Processing, vol. 47, no. 5, pp. 1421-1424, 1999.

[17] K. Doğançay, "Adaptive filtering algorithms with selective partial updates," IEEE Transactions on Circuits and Systems II: Analog and Digital Signal Processing, vol. 48, no. 8, pp. 762769, 2001.

[18] S. Werner, M. L. R. de Campos, and P. S. R. Diniz, "PartialUpdate NLMS Algorithms with Data-Selective Updating," IEEE Transactions on Signal Processing, vol. 52, no. 4, pp. 938949, 2004.

[19] M. S. E. Abadi and J. H. Husøy, "Mean-square performance of the family of adaptive filters with selective partial updates," Signal Processing, vol. 88, no. 8, pp. 2008-2018, 2008.
[20] K. Doğançay, Partial-Update Adaptive Signal Processing, Design Analysis and implementation, Academic Press, New York, NY, USA, 2009.

[21] A. W.H. Khong and P. A. Naylor, "Selective-tap adaptive filtering with performance analysis for identification of timevarying systems," IEEE Transactions on Audio, Speech and Language Processing, vol. 15, no. 5, pp. 1681-1695, 2007.

[22] S. C. Douglas, "Analysis and implementation of the maxNLMS adaptive filter," in Proceedings of the 29th Conference on Signals, Systems, and Computers, pp. 659-663, Pacific Grove, Calif, USA, October 1995.

[23] T. Aboulnasr and K. Mayyas, "Selective coefficient update of gradient-based adaptive algorithms," in Proceedings of the 1997 IEEE International Conference on Acoustics, Speech, and Signal Processing (ICASSP '97), pp. 1929-1932, Munich, Germany, April 1997.

[24] T. Schertler, "Selective block update of NLMS type algorithms," in Proceedings of the IEEE International Conference on Acoustics, Speech and Signal Processing (ICASSP'98), pp. 17171720, Seattle, Wash, USA, May 1998.

[25] A. H. Sayed, Fundamentals of Adaptive Filtering, John Wiley \& Sons, New York, NY, USA, 2003. 